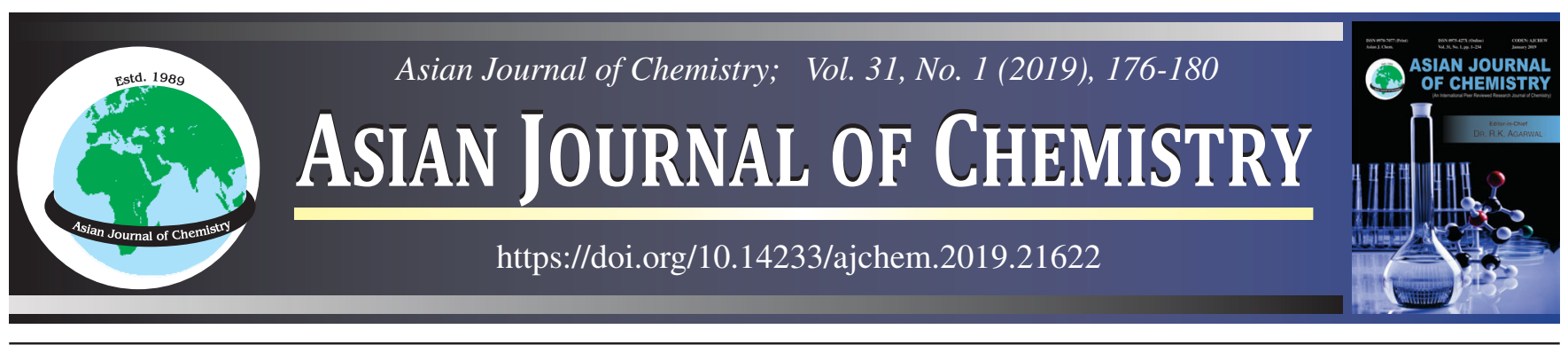

\title{
Novel Synthesis of 5-Oxo-2-thioxo-2,5-dihydro-3-thiophenecarboxylate Derivatives in Non-Aqueous Medium
}

\begin{abstract}
Nitin SRIVAStaVA* and M. SAXENA
Department of Chemistry, Amity University Uttar Pradesh, Lucknow Campus, Lucknow-226028, India

*Corresponding author: E-mail: nsrivastava1@lko.amity.edu

Received: 30 July 2018;

Accepted: 27 September 2018;

Published online: 30 November 2018;

AJC-19179

A novel synthetic method of synthesis of 5-oxo-2-thioxo-2,5-dihydro-3-thiophenecarboxylate (rhodanine) derivative is being reported using primary amines and acetylenic ester, carbon disufide and Triton-B in non-aqueous medium.

Keywords: Carbon disulfide, Primary amine, Acetylenic ester, Triton-B.

ᄂ - - - - - - - - - - - - - - - - - - - - - - - - - - -
\end{abstract}

\section{INTRODUCTION}

Rhodanine-based compounds have potential biological activities. The rhodanine derivatives have shown remarkable antifungal activity [1-4]. These compounds are also aggregators that can non-specifically interact with target proteins as well as Michael acceptors and interfere photometrically in biological assays due to their colour. They are found to inhibit targets like HCV NS3 protease [5] PMT1 mannosyltransferase [6] PRL-3 and JSP-1 phosphatase [7,8] and B lactamase [9]. After several years of research in drug discovery, they have gained a reputation as being pan assay interference compounds (PAINS) and frequent hitters in screening campaigns. Rhodaninebased compounds are also aggregators that can non-specifically interact with target proteins as well as Michael acceptors and interfere photometrically in biological assays due to their colour.

Gaikwad and Gautam [10] reported rhodanine derivatives as hypoglycemic agents which act as potent drugs to treat diabetes mellitus by lowering glucose levels in the blood. Most of them are administered orally. Shukla et al. [11] showed that substituted 3-aminomethylidene-4-thiazolidinone-2-thiones as potent antiviral agents. Classical methods for the synthesis of rhodanine is the several steps process and preparation of rhodanine structure and finally Knoevenagel condensation with aldehydes [12-16]. Taran et al. [17] proposed the synthesis of arylidene rhodanine derivatives via reaction between dithiocarbamates and aryl propiolates in the presence of $\mathrm{Bu}_{3} \mathrm{P}$ as catalyst in $\mathrm{PrOH}$. Earlier treatment of amine and its derivatives like dialkylacetylendicarboxylate and isocyante by Alizadeh et al. $[18,19]$ at room temperature led to the formation of maleimide derivatives. The reaction product was separated using ethyl acetate as organic solvent [20]. Owing to the numerous biological activity involved in the rhodanine scaffold many researchers have given various process for its synthesis [21-24].

Alizadeh et al. [25] synthesized various rhodanine derivatives via reaction of acetylene carboxylate and alkyl halides in carbon disulphide. In present study, we used carbon disulfide under similar condition for synthesis of several 5-oxo-2-thioxo2,5-dihydro-3-thiophenecarboxylate derivative (rhodanine). Carbon disulfide is preferred over common organic solvents as it is easily available, cheap and less toxic. The one pot reaction between acetylene dicarboxylate in the presence of carbon disulfide and Triton-B gave several rhodanine derivatives. The use of phase transfer catalyst Triton-B made the easier workout of the synthesized compounds with better yield.

\section{EXPERIMENTAL}

All the reagents used were of Merck, India. Identification of products was done by comparing their physical data and spectral data with the compounds already known. Infrared spectra $\left(4000-200 \mathrm{~cm}^{-1}\right)$ were analyzed on a Bomem MB-FTIR spectrophotometer while NMR spectra were recorded on Bruker Advance DPX instrument spectrophotometer (400 MHz)

This is an open access journal, and articles are distributed under the terms of the Creative Commons Attribution-NonCommercial 4.0 International (CC BY-NC 4.0) License, which allows others to copy and redistribute the material in any medium or format, remix, transform, and build upon the material, as long as appropriate credit is given and the new creations are licensed under the identical terms. 
with $\mathrm{CDCl}_{3}$ as solvent and tetramethylsilane as standard. Elemental analysis done by Carlo-Erba EA 1110 CHNOS analyzer which agreed comfortably with calculated ones. Characterization of all products was made by comparing their physical data with the reported data.

General procedure: Amine $(0.386 \mathrm{~mL})$ was added to Triton-B and stirred for $10 \mathrm{~min}$ followed by the addition of $\mathrm{CS}_{2}$ $(0.321 \mathrm{~mL})$ dropwise. Then dimethyl acetate carboxylate was added again dropwise. The mixture was stirred for $2.5 \mathrm{~h}$. TLC plate showed the formation of some new compound. The reaction was seen continuously and formation of product was monitored with TLC. After the completion, the reaction mixture was drained into distilled water and extraction was done three times using ethyl acetate (Scheme-I). IR, NMR and elemental analysis data of selected compounds are given below:

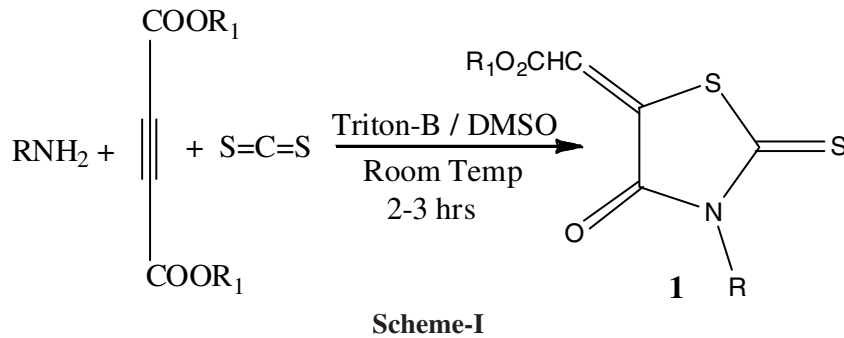

2-[4-Oxo-3-( $m$-trifluoromethylphenylmethyl)-2-thioxo1,3-thiazolidine-5-yliden]ethanoate (1): Yield $75 \%$, m.p.: $76{ }^{\circ} \mathrm{C}$, yellow powder. IR ( $\left.\mathrm{KBr}, v_{\max }, \mathrm{cm}^{-1}\right): 1720(\mathrm{C}=\mathrm{O}), 1680$ $(\mathrm{C}=\mathrm{C}), 1342,1187(\mathrm{C}=\mathrm{S}) .{ }^{1} \mathrm{H}$ NMR: $\left(400 \mathrm{MHz}, \mathrm{CDCl}_{3}\right): \delta 7.5$ (multiplet arom. $\mathrm{H}$ ), 6.8-7.0 (singlet $\delta$ vinylic $\mathrm{H}$ ), $\delta 5.4$ (singlet $2 \mathrm{H}$ adj. to $\mathrm{N}$ ), 3.9 (singlet $3 \mathrm{H}$ of $\mathrm{CH}_{3} \mathrm{O}$ ). Elemental analysis calcd. (found) $\%$ for $\mathrm{C}_{14} \mathrm{H}_{10} \mathrm{NO}_{3} \mathrm{~S}_{2} \mathrm{~F}_{3}$ : C 47.9 (46.8), H 2.84 (2.64), O 13.6 (14.4), S 18.2 (17.2), F 16.2 (15.9).

2-[4-Oxo-3-( $p$-trifluoromethylphenylmethyl)-2-thioxo1,3-thiazolidine-5-yliden]ethanoate (2): Yield $77 \%$, m.p.: $82^{\circ} \mathrm{C}$, yellow powder. IR $\left(\mathrm{KBr}, v_{\max }, \mathrm{cm}^{-1}\right): 1720(\mathrm{C}=\mathrm{O}), 1683$ $(\mathrm{C}=\mathrm{C}), 1330,1187(\mathrm{C}=\mathrm{S}) .{ }^{1} \mathrm{H}$ NMR: $\left(400 \mathrm{MHz}, \mathrm{CDCl}_{3}\right): \delta 7.5$ (multiplet arom. $\mathrm{H}$ ), 6.8-7.0 (singlet $\delta$ vinylic $\mathrm{H}$ ), $\delta 5.4$ (singlet $2 \mathrm{H}$ adj. to $\mathrm{N}$ ), 3.9 (singlet $3 \mathrm{H}$ of $\mathrm{CH}_{3} \mathrm{O}$ ). Elemental analysis calcd. (found) $\%$ for $\mathrm{C}_{14} \mathrm{H}_{10} \mathrm{NO}_{3} \mathrm{~S}_{2} \mathrm{~F}_{3}$ : C 47.8 (47.6), $\mathrm{H} 2.90$ (2.98), O 13.70 (13.86), S 18.0 (18.25), F 16.6 (17.6).

2-[4-Oxo-3-(propylphenylmethyl)-2-thioxo-1,3-thiazolidine-5-yliden]ethanoate (3): Yield $80 \%$, m.p.: $128^{\circ} \mathrm{C}$, yellow powder. IR (KBr, $\left.v_{\max }, \mathrm{cm}^{-1}\right): 1725(\mathrm{C}=\mathrm{O}), 1685(\mathrm{C}=\mathrm{C}), 1330$,
1187 (C=S). ${ }^{1} \mathrm{H}$ NMR: (400 MHz, $\left.\mathrm{CDCl}_{3}\right): \delta 4.5$ (2H arom.), $\delta$ 4.0 (singlet $\mathrm{OCH}_{3}$ ), $\delta 3.0$ (2H arom.), $\delta 2.0$ ( $4 \mathrm{H}$ multiplet), $\delta$ 1.9 (1H singlet). Elemental analysis calcd. (found) $\%$ for $\mathrm{C}_{15} \mathrm{H}_{15} \mathrm{NO}_{3} \mathrm{~S}_{2}$ : C 56.07 (55.20), H 4.6 (4.8), N 4.36 (4.10), O 14.9 (14.2), S 19.9 (20.2).

2-[4-Oxo-3-(1'-methylethyl)-2-thioxo-1,3-thiazolidine5-yliden]ethanoate (4): Yield $80 \%$, m.p.: $12{ }^{\circ} \mathrm{C}$, yellow oil. IR $\left(\mathrm{KBr}, v_{\max }, \mathrm{cm}^{-1}\right)$ : $1725(\mathrm{C}=\mathrm{O}), 1670(\mathrm{C}=\mathrm{C}), 1315,1187$ $(\mathrm{C}=\mathrm{S}) .{ }^{1} \mathrm{H}$ NMR: (400 MHz, $\left.\mathrm{CDCl}_{3}\right): \delta 1.54$ (6H doublet), $\delta$ 3.88.0 (3H singlet $\left.\mathrm{OCH}_{3}\right), \delta 5.20\left(1 \mathrm{H}\right.$ septet $\left.\mathrm{CH}-\mathrm{CH}_{3}\right), \delta 6.77$ $(1 \mathrm{H}$, singlet ethylenic $\mathrm{H}), \delta 1.9(1 \mathrm{H}$ singlet). Elemental analysis calcd. (found) $\%$ for $\mathrm{C}_{9} \mathrm{H}_{11} \mathrm{NO}_{2} \mathrm{~S}_{2}$ : C 44.08 (46.4), $\mathrm{H} 4.48$ (5.02), N 5.71 (6.24), O 15.59 (14.8), S 26.12 (27.02).

2-[4-Oxo-3-(2'-methylpropyl)-2-thioxo-1,3-thiazolidine-5-yliden]ethanoate (5): Yield $80 \%$, m.p.: $8{ }^{\circ} \mathrm{C}$, pale yellow oil. IR $\left(\mathrm{KBr}, v_{\max }, \mathrm{cm}^{-1}\right)$ : $1715(\mathrm{C}=\mathrm{O}), 1690(\mathrm{C}=\mathrm{C}), 1315$, $1187(\mathrm{C}=\mathrm{S}) .{ }^{1} \mathrm{H}$ NMR $\left(400 \mathrm{MHz}, \mathrm{CDCl}_{3}\right): \delta 2.25-2.30(1 \mathrm{H}$ multiplet $\mathrm{CH}_{2} \mathrm{CH}-\mathrm{CH}_{3}$ ), $\delta 3.87$ (3H singlet $\left.\mathrm{OCH}_{3}\right), \delta 3.93[2 \mathrm{H}$ doublet $\mathrm{CH}_{2} \mathrm{CH}-\left(\mathrm{CH}_{3}\right)_{2}$ ]. Elemental analysis calcd. (found) $\%$ for $\mathrm{C}_{10} \mathrm{H}_{13} \mathrm{NO}_{3} \mathrm{~S}_{2}$ : C 46.66 (45.86), $\mathrm{H} 4.28$ (4.04), N 5.44 (5.24), O 18.67 (19.07), S 24.90 (25.20).

\section{RESULTS AND DISCUSSION}

One pot synthesis of rhodanine derivatives having coveted biological and pharmacological activities using Triton-B, carbon disulfide at room temperature is reported. The reaction of carbon disulfide with several amines in presence of dialkylacetylenedicarboxylate was spontaneous at room temperature in DMSO/ Triton-B. Also these reactions were completed in 2-3 h (Table-1).

The synthesized compounds formed were identified by their elemental analysis, IR and ${ }^{1} \mathrm{H}$ NMR spectra. The ${ }^{1} \mathrm{H}$ NMR spectra of compound 1 exhibited 3 sharp singlets which were thought to be due to $\mathrm{OCH}_{3}$ group $(\delta=3.9)$, methylene protons $(\delta=5.32)$ and vinylic proton at $(\delta=6.85)$. The phenyl group gave characteristic aromatic signals in the NMR spectrum.

A possible mechanism of the reaction between carbon disulfide and amine in the presence of Triton-B is being put forward in Scheme-II. Compound $\mathbf{1}$ could results from initial addition of carbon disulfide to amine and subsequent attack of alkylammoniumcarbothioate on acetylenic ester to yield intermediate. Cyclization of intermediate and subsequent loss of $\mathrm{R}_{1} \mathrm{OH}$ lead to the formation of compound $\mathbf{1}$ (Scheme-II).

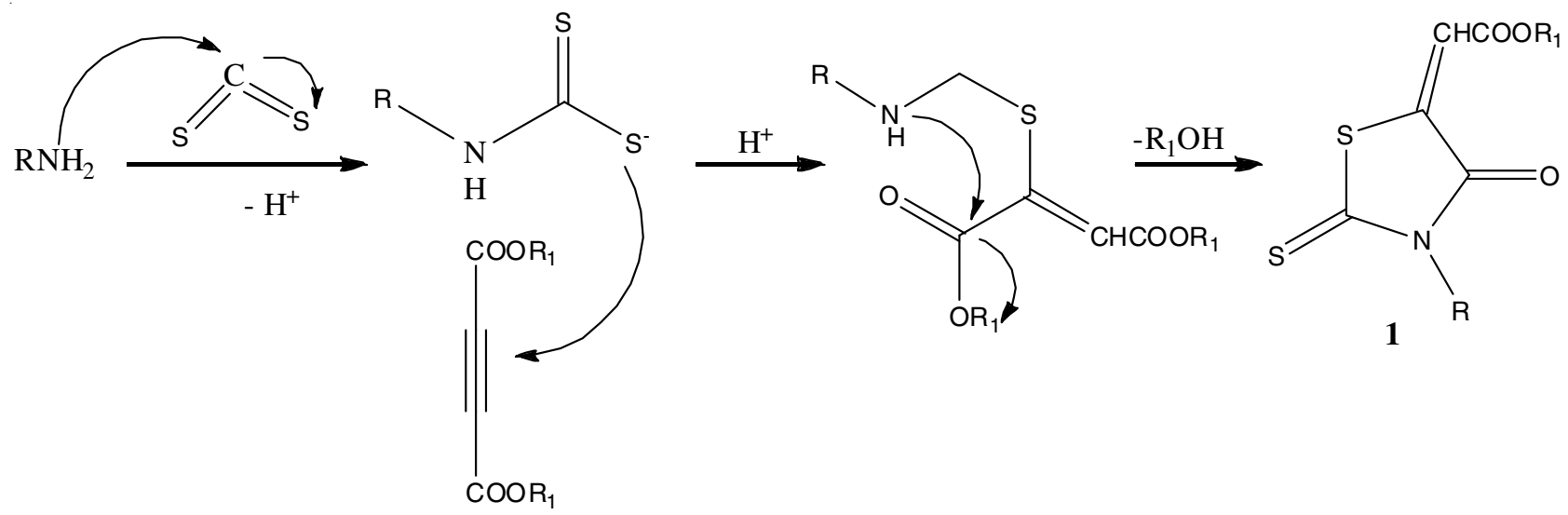


178 Srivastava et al.

Asian J. Chem.

TABLE-1

S. No.

Amine used

$\mathrm{R}_{1}$<smiles>NCc1cccc(C(F)(F)F)c1</smiles><smiles>CC</smiles>

2<smiles>NCc1ccc(C(F)(F)F)cc1</smiles><smiles>COC(=O)C=C1SC(=S)N(Cc2ccc(C(F)(F)F)cc2)C1=O</smiles>

3<smiles>NCCc1ccccc1</smiles>

4<smiles>CC(C)N</smiles>

5<smiles>CC(C)CN</smiles>

6<smiles>NC1CCCC1</smiles>

Structure of rhodamine derivatives

Yield (\%)<smiles>COC(OC)=C1SC(=S)N(Cc2cccc(C(F)(F)F)c2)C1=O</smiles>

71

77

80

80<smiles>COC(C)=C1SC(=S)N(CC(C)C)C1=O</smiles>

80<smiles>COC(OC)=C1SC(=S)N(C2CCCC2)C1=O</smiles>

76 


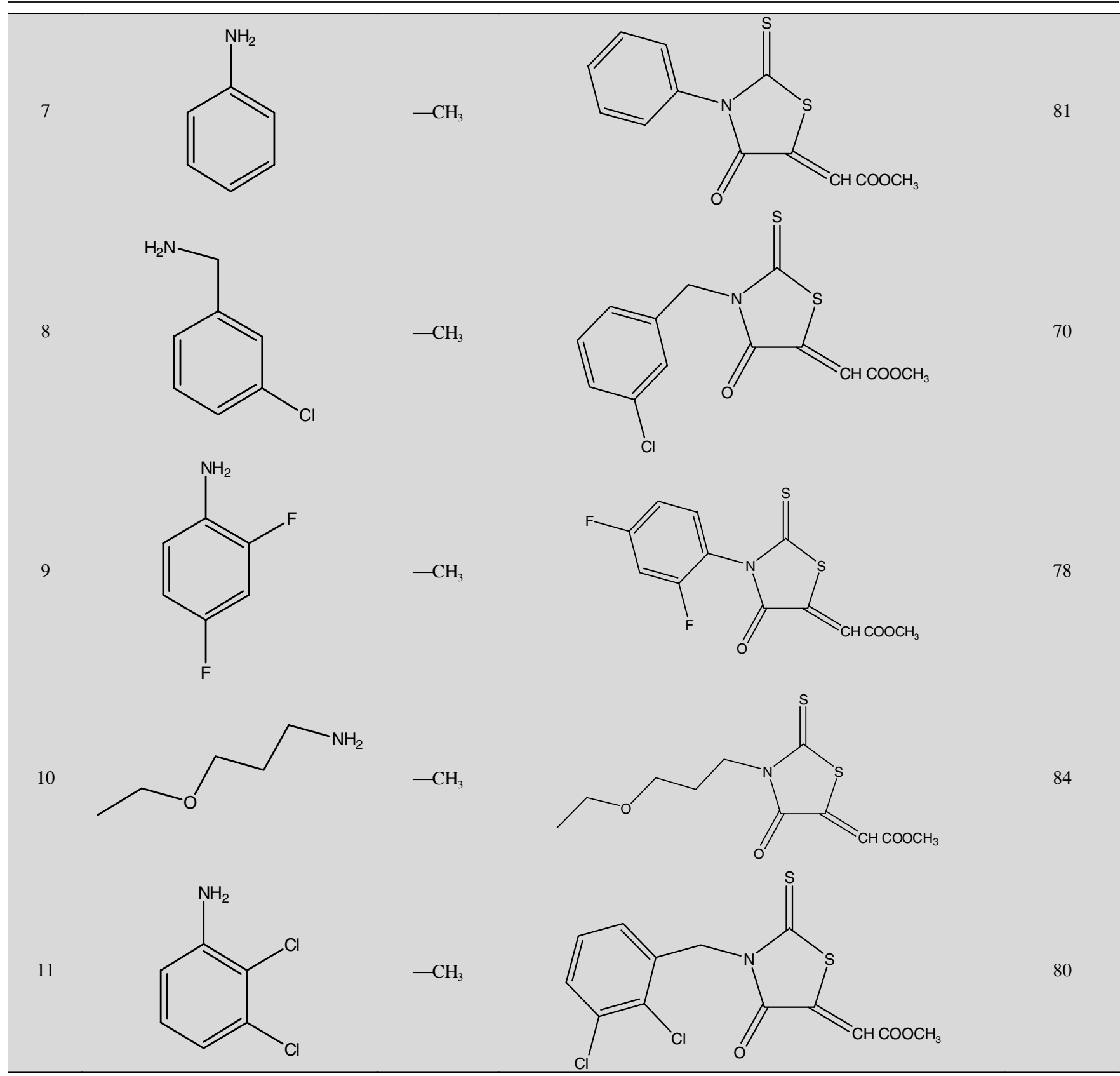

\section{Conclusion}

In conclusion, carbon disulfide mediated synthesis has been reported for the synthesis of rhodanine derivatives and offers some notable and distinct advantages over usually employed procedure such as simplicity, room temperature conditions, simple work-up, high yield and cheap reagent.

\section{ACKNOWLEDGEMENTS}

The authors gratefully acknowledge fruitful discussions with Dr. Devdutt Chaturvedi regarding the research and Amity University Uttar Pradesh Lucknow Campus, India for providing the infrastructure to conduct the research.

\section{CONFLICT OF INTEREST}

The authors declare that there is no conflict of interests regarding the publication of this article.

\section{REFERENCES}

1. Y. Inamori, Y. Okamoto, Y. Takegawa, H. Tsujibo, Y. Sakagami, Y. Kumeda, M. Shibata and A. Numata, Biosci. Biotechnol. Biochem., 62, 1025 (1998); https://doi.org/10.1271/bbb.62.1025.

2. J. Miao, C.-J. Zheng, L.-P. Sun, M.-X. Song, L.-L. Xu and H.-R. Piao, Med. Chem. Res., 22, 4125 (2013);

https://doi.org/10.1007/s00044-012-0417-z.

3. P. Mitra and A.S. Mitra, J. Indian Chem. Soc., 61, 77 (1984).

4. A.A. Khalek, C.R. Ashby Jr., B.A. Patel, T.T. Talele and M.N. Seleem, PLoS One, 11, e0164227 (2016); https://doi.org/10.1371/journal.pone.0164227.

5. W. Sing, C.L. Lee, S.L. Yeo, S.P. Lim and M.M. Sim, Bioorg. Med. Chem. Lett., 11, 91 (2001);

https://doi.org/10.1016/S0960-894X(00)00610-7.

6. M.G. Orchard, J.C. Neuss, C.M. Galley, A. Carr, D.W. Porter, P. Smith, D.I.C. Scopes, D. Haydon, K. Vousden, C.R. Stubberfield, K. Young and M. Page, Bioorg. Med. Chem. Lett., 14, 3975 (2004); https://doi.org/10.1016/j.bmcl.2004.05.050. 
7. N.S. Cutshall, C. O' Day and M. Prezhdo, Bioorg. Med. Chem. Lett., 15, 3374 (2005); https://doi.org/10.1016/j.bmcl.2005.05.034.

8. J.H. Ahn, S.J. Kim, W.S. Park, S.Y. Cho, J.D. Ha, S.S. Kim, S.K. Kang, D.G. Jeong, S.-K. Jung, S.-H. Lee, H.M. Kim, S.K. Park, K.H. Lee, C.W. Lee, S.E. Ryu and J.-K. Choi, Bioorg. Med. Chem. Lett., 16, 2996 (2006); https://doi.org/10.1016/j.bmcl.2006.02.060.

9. E.B. Grant, D. Guiadeen, E.Z. Baum, B.D. Foleno, D.A. Montenegro, H. Jin, E.A. Nelson, K. Bush and D.J. Hlasta, Bioorg. Med. Chem. Lett., 10, 2179 (2000); https://doi.org/10.1016/S0960-894X(00)00444-3.

10. N.J. Gaikwad and P. Gautam, Indian J. Heterocycl. Chem., 12, 181 (2002).

11. S.K. Shukla, S.P. Singh, L.P. Awasthi and D.D. Mukherjee, Indian J. Pharm. Sci., 44, 153 (1982).

12. B.B. Lohray, V. Bhushan, P.B. Rao, G.R. Madhavan, N. Murali, K.N. Rao, G.R. Madhavan, N. Murali, K.N. Rao, K. Anantha Reddy, B.M. Rajesh, P. Ganpathy Reddy, R. Chakrabarti and R. Rajagopalan, Bioorg. Med. Chem. Lett., 7, 785 (1997); https://doi.org/10.1016/S0960-894X(97)00118-2.

13. S.P. Singh, S.S. Parmar, K. Raman and V.I. Stenberg, Chem. Rev., 81, 175 (1981); https://doi.org/10.1021/cr00042a003.

14. C.L. Lee and M.M. Sim, Tetrahedron Lett., 41, 5729 (2000); https://doi.org/10.1016/S0040-4039(00)00866-2.

15. F.C. Brown, Chem. Rev., 61, 463 (1961); https://doi.org/10.1021/cr60213a002.
16. W.-D. Rudorf and R. Schwarz, Hetrocycles, 24, 3459 (1986); https://doi.org/10.3987/R-1986-12-3459.

17. S. Gabillet, D. Lecercle, O. Loreau, M. Carboni, S. Dezard, J. Gomis and M. Taran, Org. Lett., 9, 3925 (2007); https://doi.org/10.1021/ol701563e.

18. A. Alizadeh, F. Movahedi, H. Masrouri and L.-G. Zhu, Synthesis, 3431 (2006); https://doi.org/10.1055/s-2006-950234.

19. A. Alizadeh, F. Movahedi and A.A. Esmaili, Tetrahedron Lett., 47, 4469 (2006); https://doi.org/10.1016/j.tetlet.2006.04.040.

20. J. Liu, Y. Zhou, Y. Wu, X. Li and A.S.C. Chan, Tetrahedron Asymm., 19, 832 (2008); https://doi.org/10.1016/..tetasy.2008.03.015.

21. D. Kaminskyy, A. Kryshchyshyn and R. Lesyk, Expert Opin. Drug Discov., 12, 1233 (2017); https://doi.org/10.1080/17460441.2017.1388370.

22. S.S. Alneyadi, Heterocycles, 96, 803 (2018); https://doi.org/10.3987/REV-17-878.

23. T. Tomasic and L.P. Masiè, Expert Opin. Drug Discov., 7, 549 (2012); https://doi.org/10.1517/17460441.2012.688743.

24. T. Tomasic and L.P. Masiè, Curr. Med. Chem., 16, 1596 (2009); https://doi.org/10.2174/092986709788186200.

25. A. Alizadeh, S. Rostamnia, N. Zohreh and R. Hosseinpour, Tetrahedron Lett., 50, 1533 (2009); https://doi.org/10.1016/j.tetlet.2008.12.107. 